\title{
RESULTADOS PERINATALES SEGÚN LA VÍA DEL PARTO EN PRETÉRMINOS EN LA CLÍNICA MATERNIDAD RAFAEL CALVO
}

\author{
PERINATAL OUTCOMES IN RELATION TO MODE OF DELIVERY IN \\ PRETERM INFANTS IN CLÍNICA MATERNIDAD RAFAEL CALVO
}

\author{
Beracaza-Robledo Cristina ${ }^{1}$ \\ Salcedo-Ramos Francisco ${ }^{2}$ \\ Rodríguez-Yance Benjamín ${ }^{2}$ \\ Castañeda-Alfonso Sergio ${ }^{3}$ \\ Olarte-Parra Juan ${ }^{3}$
}

Correspondencias: crisber8@hotmail.com

Recibido para evaluación: octubre - 15 - 2014; aceptado para publicación: agosto - 20 - 2015

\section{RESUMEN}

Introducción: el parto pretérmino (PP) actualmente constituye un problema de salud pública. El aumento de las tasas de prematuridad ha llevado al aumento en las tasas de complicaciones neonatales, inherentes a la falta de madurez fetal.

Objetivo: observar los resultados perinatales logrados en nacidos pretérminos (NP) en un centro obstétrico de referencia.

Materiales y métodos: estudio descriptivo-retrospectivo en el que se determinó la prevalencia de las complicaciones neonatales en NP, teniendo en cuenta la vía del parto. Realizado en la ESE Clínica Maternidad Rafael Calvo durante el período comprendido entre el 1 de enero y el 31 de diciembre de 2014.

Resultados: se incluyeron 822 historias clínicas de pacientes, 426 (51.8\%) que tuvieron parto por cesárea y $396(48.2 \%)$ por vía vaginal, la mediana de edad de los grupos por cesárea y vaginal fue de 23 y 20 años, respectivamente. Dentro de los diagnósticos de ingreso resalta de manera significativa, mayor ocurrencia en el grupo cesárea del oligohidramnios y los trastornos hipertensivos del embarazo, mientras que el trabajo de PP de inicio espontáneo fue más frecuente en el grupo de parto vaginal, $\mathrm{p}<0.0001$. No hubo diferencia estadísticamente significativa en la presentación de hemorragia intraventricular, trauma neonatal, enterocolitis necrotizante y muerte neonatal entre los dos grupos. Sin embargo, se presentaron diferencias en la infección neonatal, ingreso a UCI, requerimiento de ventilación mecánica y tiempo de ventilación, siendo mayor en el grupo de parto por cesárea.

Conclusión: los resultados perinatales adversos más prevalentes en NP por cesárea fueron: necesidad de oxígeno suplementario, síndrome de distrés respiratorio, ingreso a UCIN, infección neonatal, sepsis temprana, taquipnea transitoria y la enfermedad de membrana hialina. En NP por vía vaginal las morbilidades más prevalentes observadas fueron: distrés respiratorio, taquipnea transitoria, sepsis e infección neonatal. Rev. cienc.biomed. 2015;6(2):282-289

\section{PALABRAS CLAVE}

Cesárea; Morbilidad; Mortalidad; Parto pretérmino

\footnotetext{
Médico. Estudiante de Postgrado. Ginecología y Obstetricia. Facultad de Medicina. Universidad de Cartagena. Cartagena. Colombia Médico. Especialista en Ginecología y Obstetricia. Docente Departamento de Ginecología y Obstetricia. Facultad de Medicina. Universidad de Cartagena. Cartagena. Colombia.

3 Estudiante de Medicina. Facultad de Medicina. Universidad de Cartagena. Colombia
} 
ISSN: 2215-7840, 6(2), julio-diciembre 2015, Beracaza-Robledo Cristina, Salcedo-Ramos Francisco, Rodríguez-Yance Benjamín, Castañeda-Alfonso Sergio, Olarte-Parra Juan

\section{SUMMARY}

Introduction: preterm birth (PB) is now a public health problem, as the increased rates of prematurity has led to a gradual increase in rates, inherent lack of fetal maturity neonatal complications.

Objective: to observe perinatal outcomes achieved in preterm infants (PI) in an obstetric referral center.

Methods: descriptive-retrospective study in which was determined the prevalence of neonatal complications in (PI), keeping in mind the mode of delivery. The study was carried out in Clínica Maternidad Rafael Calvo in the period between 1 January and 31 December 2014.

Results: in total 822 patients, 425 of them with cesarean delivery and 396 with vaginal birth were included, the average age of cesarean and vaginal groups was 23 and 20 years, respectively. Among the highlights admission diagnoses significantly higher occurrence in the cesarean group of oligohydramnios, and hypertensive disorders of pregnancy, while preterm obstetric labor (was more frequent in the vaginal delivery group, $p$ $<0.0001)$. There was not significant difference in the presentation of intraventricular hemorrhage, neonatal trauma, neonatal necrotizing enterocolitis and death between the two groups. However, there were differences in the presentation of neonatal infection, ICU admission, requirement for mechanical ventilation and mechanical ventilation time, being higher in the cesarean group.

Conclusions: the most prevalent adverse perinatal outcomes in PI born by cesarean delivery were: necessity of supplementary oxygen, infant respiratory distress syndrome, admission to neonatal intensive care unit, neonatal infection, early sepsis, transient tachypnea and hyaline membrane disease. In the PI born by vaginal delivery the most prevalent morbidities were: respiratory distress, transient tachypnea, sepsis and neonatal infection. Rev.cienc.biomed. 2015; 6(2):282-289

\section{KEYWORDS}

Cesarean section; Morbidity; Mortality, Preterm birth

\section{INTRODUCCIÓN}

El parto pretérmino (PP) se define como aquel parto que sucede antes de las 37 semanas completas o 259 días de gestación. Es determinante e importante para la mortalidad y morbilidad neonatal, con consecuencias adversas para la salud especialmente por la mayor tasa de parálisis cerebral, déficit sensorial y dificultad de aprendizaje, así como de enfermedades respiratorias, en comparación con niños nacidos a término (1).

La morbilidad asociada con el PP a menudo se extiende a la vida posterior ocasionando enormes limitaciones físicas, psicológicas y elevados costos económicos tanto para la familia como para el sistema de salud, disminuyendo la calidad de vida de los pacientes $(2,3)$. Durante varias décadas, los nacimientos pretérminos han sido foco de la investigación y de intervención en salud pública, a pesar de ello, la tasa de incidencia se ha mantenido estable e incluso se ha incrementado (4).

El aumento de PP a nivel mundial, sobre todo en África, Asia y América Latina (4) justifican que sea considerado un importante problema de salud perinatal. Si bien los países en desarrollo son los que sufren la carga más alta de PP en términos absolutos, en América del Norte también se observa tasa elevada. Es necesario comprender mejor las causas de la prematuridad y tener estimaciones más precisas de la problemática en cada país $(4,5)$.

El PP se divide en subcategorías según la edad gestacional de nacimiento y las semanas cursadas, de la siguiente manera: [A] pretérmino extremo, antes de las 28 semanas, [B] muy pretérmino, entre las 28 y 32 semanas, [C] pretérmino moderado, $33 \mathrm{se-}$ manas, [D] pretérmino tardío de 34 a 36 semanas y 6 días. También se puede dividir la prematuridad como espontánea o inducida y a su vez, los nacimientos espontáneos pueden ser con membranas íntegras o rotas (6).

La especialización e infraestructura hospitalaria influyen en los resultados perinatales esperados del manejo de los nacidos pretérmino. Estudios sugieren que la mortalidad por debajo de las 32 semanas disminuye si 
el parto es atendido en un centro con disponibilidad de atención perinatal especializada (7-9).

Un importante debate en el PP es la vía del parto. Es usual la disyuntiva para decidir entre realizar cesárea o brindar parto vaginal. Se asevera que en los casos donde no existen indicaciones para cesárea, el nacido pretérmino podría beneficiarse del parto vaginal por las menores tasas de complicaciones neonatales, lo cual se traduce en mejores resultados perinatales $(7,8)$. No suele aceptarse la recomendación de practicar cesárea de rutina con la finalidad de mejorar la supervivencia o disminuir la morbilidad en los prematuros extremos (10). El objetivo fue observar los resultados perinatales logrados en nacidos pretérmino (NP) en un centro obstétrico de referencia.

\section{MATERIALES Y MÉTODO}

Estudio descriptivo-retrospectivo para determinar la prevalencia de complicaciones neonatales teniendo en cuenta la vía del parto. Se adelantó revisión de las historias clínicas y se sentó la información obtenida en una base de datos. La población de estudio fueron las historias clínicas de las pacientes embarazadas que tuvieron PP por cesárea o vaginal, así como la historia clínica de sus neonatos que fueron atendidos en la Clínica de Maternidad Rafael Calvo, en Cartagena-Colombia, entre enero y diciembre del 2014.

Los criterios de inclusión fueron todas las historias clínicas de las pacientes que presentaron parto vaginal o cesárea con embarazo único antes de las 37 semanas de gestación. Se excluyeron las historias clínicas de aquellas pacientes cuyos recién nacidos fueron remitidos a unidad de cuidados intensivos de otra institución, hubiesen presentado malformaciones congénitas o diagnosticados con síndromes genéticos.

Las variables de estudios se dividieron en tres grupos: [A] aspectos sociodemográficos, [B] variables neonatales, [C] maternas. Para el análisis se utilizó el software estadístico Epi-Info 7. Se hallaron medidas de tendencia central para las variables sociode- mográficas, mediante tablas de contingencia y prueba del Chi cuadrado, se describieron las relaciones existentes entre los resultados neonatales y la vía del parto. Estudio sin implicaciones éticas realizado anónimamente en documentos, por ser retrospectivo no tuvo impacto la decisión clínica del manejo de las pacientes.

\section{RESULTADOS}

Se consideraron adecuadas para el estudio 822 historias clínicas de gestantes con feto único que tuvieron PP. El 51.8\% (426) de los recién nacidos fueron por cesárea y el 48.2\% (396) por vía vaginal. La mediana de edad de los grupos de parto por cesárea y vaginal fue de 23 y 20 años, respectivamente. El $80 \%$ de ambos grupos fueron procedentes de Cartagena. Los diagnósticos de ingreso de mayor ocurrencia en el grupo cesárea fueron: oligoamnios, preeclampsia, preeclampsia severa y trastornos hipertensivos del embarazo, mientras que el más frecuente en el grupo que tuvo parto vaginal fue: trabajo de PP de inicio espontáneo, $\mathrm{p}<0.0001$. La presentación cefálica fue más frecuente en la vía vaginal mientras que la podálica lo fue en el grupo cesárea, $\mathrm{p}$ $<0.0001$. (Tabla N¹).

Al comparar la frecuencia de eventos neonatales por vía del parto se encontró mayor necesidad de oxígeno suplementario, síndrome de distrés respiratorio, infección neonatal temprana y necesidad de manejo en unidad de cuidados intensivos en el grupo de nacidos por cesárea en comparación con el grupo vaginal, $p<0.0001$. No hubo diferencias estadísticamente significativas en la frecuencia de trauma neonatal (Tabla N02).

Entre los neonatos con síndrome de dificultad respiratoria se encontró mayor frecuencia de enfermedad de membrana hialina en los nacido por cesárea, $4.5 \%$ comparado con $1.3 \%$ en los nacidos vía vaginal, $\mathrm{p}=0.0125$. De los neonatos que requirieron manejo en unidad de cuidados intensivos neonatal, el $12 \%$ de los del grupo de cesárea y el $4 \%$ de vía vaginal requirieron ventilación mecánica, teniendo mediana de necesidad de tres días en el primer grupo [RI:2-6] y de dos en el segundo [RI:1-3]. 
ISSN: 2215-7840, 6(2), julio-diciembre 2015, Beracaza-Robledo Cristina, Salcedo-Ramos Francisco, Rodríguez-Yance Benjamín,

\begin{tabular}{|c|c|c|c|}
\hline \multicolumn{4}{|c|}{$\begin{array}{c}\text { TABLA No } 1 . \\
\text { CARACTERISTÍCAS DE LA POBLACIÓN ESTUDIADA } \\
n=822\end{array}$} \\
\hline & $\begin{array}{l}\text { Cesárea } \\
\mathrm{N}=426\end{array}$ & $\begin{array}{l}\text { Vaginal } \\
\mathrm{N}=396\end{array}$ & Valor $\mathrm{p}$ \\
\hline Edad Me [RI] & $23[20-30]$ & $20[17-25]$ & $<0.0001$ \\
\hline Procedencia Cartagena & $342(80.5)$ & $328(82.8)$ & 0.4609 \\
\hline \multicolumn{4}{|c|}{ Diagnóstico de ingreso $\mathrm{n}(\%)$} \\
\hline Eclampsia & $1(0.2)$ & - & 0.8029 \\
\hline Estado fetal no satisfactorio & $5(1.2)$ & - & 0.0876 \\
\hline Oligohidramnios & $12(2.8)$ & $1(0.2)$ & 0.0070 \\
\hline Preeclampsia & $21(4.9)$ & $2(0.5)$ & 0.0004 \\
\hline Preeclampsia severa & $82(10.3)$ & $5(1.3)$ & $<0.0001$ \\
\hline $\begin{array}{l}\text { Restricción crecimiento } \\
\text { intrauterino }\end{array}$ & $2(0.5)$ & $0(0.0)$ & 0.4952 \\
\hline $\begin{array}{l}\text { Ruptura prematura de } \\
\text { membranas }\end{array}$ & $94(22.2)$ & $107(27.0)$ & 0.1407 \\
\hline $\begin{array}{l}\text { Trastorno hipertensivo del } \\
\text { embarazo }\end{array}$ & $42(9.9)$ & $6(1.2)$ & $<0.0001$ \\
\hline Trabajo de parto pretérmino & $105(24.7)$ & $219(55.3)$ & $<0.0001$ \\
\hline Diferentes diagnósticos & $61(14.3)$ & $56(14.1)$ & 0.9820 \\
\hline \multicolumn{4}{|c|}{ Presentación fetal n (\%) } \\
\hline Cefálico & $364(86.3)$ & $381(96.7)$ & $<0.0001$ \\
\hline Podálico & $52(12.3)$ & $12(3.0)$ & $<0.0001$ \\
\hline Transverso & $6(1.4)$ & $1(0.2)$ & 0.1387 \\
\hline \multicolumn{4}{|c|}{ Complicaciones n (\%) } \\
\hline Hemorragia & $1(0.2)$ & $3(0.7)$ & 0.6116 \\
\hline Endometritis & $0(0.0)$ & $1(0.2)$ & 0.8034 \\
\hline Necesidad de legrado uterino & $2(0.5)$ & $6(1.5)$ & 0.2970 \\
\hline Reintervención operatoria & $2(0.5)$ & $1(0.5)$ & 0.6117 \\
\hline Datos confusos & $1(0.2)$ & $6(1.5)$ & 0.1128 \\
\hline Diversas complicaciones & $10(2.3)$ & $6(1.5)$ & 0.5781 \\
\hline
\end{tabular}

\begin{tabular}{|c|c|c|c|}
\hline \multicolumn{4}{|c|}{$\begin{array}{c}\text { TABLA NO } 2 . \\
\text { RESULTADOS PERINATALES } \\
n=822\end{array}$} \\
\hline & $\begin{array}{l}\text { Cesárea } \\
\mathrm{N}=426\end{array}$ & $\begin{array}{l}\text { Vaginal } \\
\mathrm{N}=396\end{array}$ & Valor p \\
\hline Reanimación & $35(8.2)$ & $25(6.3)$ & 0.3615 \\
\hline Oxígeno suplementario & $269(63.3)$ & $162(40.9)$ & $<0.0001$ \\
\hline Síndrome distrés respiratorio & $265(62.3)$ & $161(40.7)$ & $<0.0001$ \\
\hline $\begin{array}{l}\text { Taquipnea transitoria del recién } \\
\text { nacido }\end{array}$ & $56(13.2)$ & $68(17.2)$ & 0.1334 \\
\hline $\begin{array}{l}\text { Enfermedad de membrana } \\
\text { hialina }\end{array}$ & $19(4.5)$ & $5(1.3)$ & 0.0125 \\
\hline Hemorragia intraventricular & $2(0.5)$ & $1(0.2)$ & 0.9179 \\
\hline Trauma obstétrico neonatal & $2(0.7)$ & $4(1.5)$ & 0.4670 \\
\hline Cefalohematoma & $0(0.0)$ & $1(0.2)$ & 0.8395 \\
\hline Equimosis & $0(0.0)$ & $2(0.5)$ & 0.4547 \\
\hline Fractura de clavícula & $0(0.0)$ & $1(0.2)$ & 0.8395 \\
\hline Fractura de fémur & $1(0.2)$ & $0(0.0)$ & 0.4344 \\
\hline Edema frontal & $1(0.2)$ & $0(0.0)$ & 0.4344 \\
\hline Enterocolitis necrotizante & $9(2.1)$ & $4(1.0)$ & 0.3240 \\
\hline Infección neonatal & $125(29.4)$ & $45(11.4)$ & $<0.0001$ \\
\hline Sepsis temprana & $106(24.9)$ & $43(10.9)$ & $<0.0001$ \\
\hline Bronconeumonía temprana & $44(10.4)$ & $13(3.3)$ & 0.0001 \\
\hline UCIN & $225(52.9)$ & $112(28.3)$ & $<0.0001$ \\
\hline Ventilación mecánica & $51(12.0)$ & $16(4.0)$ & $<0.0001$ \\
\hline Días Me [RI] & $3[2-6]$ & $2[1-3]$ & $<0.0001$ \\
\hline Muerte neonatal & $17(4.0)$ & $18(4.5)$ & 0.8556 \\
\hline Sepsis severa & $6(1.4)$ & $2(0.5)$ & 0.3368 \\
\hline Shock séptico & $4(0.9)$ & $0(0.0)$ & 0.1678 \\
\hline Disfunción orgánica múltiple & $12(2.8)$ & $7(1.8)$ & 0.4719 \\
\hline $\begin{array}{l}\text { Coagulopatía intravascular } \\
\text { diseminada }\end{array}$ & $5(1.2)$ & $1(0.2)$ & 0.1997 \\
\hline
\end{tabular}

Se observó diferencia estadísticamente significativa en la necesidad de ventilación mecánica y en la duración de la ventilación mecánica, $p<0.0001$, siendo menor en el parto vaginal. Las causas de muerte neonatal fueron en orden de frecuencia: disfunción orgánica múltiple, sepsis severa, coagulación intravascular diseminada y shock séptico. No se observó diferencia entre los grupos de estudio.

La Tabla $\mathrm{N}^{\circ} 3$ presenta los requerimientos de oxígeno y reanimación acorde a la clasificación de la condición de prematuridad, además, lo referente a complicaciones respiratorias y hemorrágicas. No fue diferente la presencia de trauma obstétrico neonatal pero sí la tasa de mortalidad neonatal, la cual fue del $85 \%$ en pretérminos extremos, significativamente mucho más alta a la de los otros grupos.

\section{DISCUSIÓN}

La incidencia de PP es variable, aunque es un evento de distribución global, es más alta en África y el sur de Asia. En el año 2005 se registraron 12.9 millones de $\mathrm{PP}$, lo que representa el $9.6 \%$ de todos los nacimientos a nivel mundial. Aproximadamente 11 millones $(85 \%)$ de ellos se concentraron en África y Asia, mientras que en Europa y América del Norte (excluido México) se registraron 0.5 millones en cada región geográfica. En América Latina y en el Caribe fueron 0.9 millones $(4,11)$. 


\begin{tabular}{|c|c|c|c|c|}
\hline \multicolumn{5}{|c|}{$\begin{array}{l}\text { TABLA No } 3 . \\
\text { RESULTADOS PERINATALES SEGÚN EDAD GESTACIONAL } \\
n=822\end{array}$} \\
\hline & $\begin{array}{c}\text { Pretérmino } \\
\text { extremo } \\
\mathrm{N}=7\end{array}$ & $\begin{array}{l}\text { Muy } \\
\text { pretérmino } \\
\mathrm{N}=67\end{array}$ & $\begin{array}{l}\text { Pretérmino } \\
\text { intermedio } \\
\mathrm{N}=99\end{array}$ & $\begin{array}{l}\text { Pretérmino } \\
\text { tardío } \\
\mathrm{N}=649\end{array}$ \\
\hline Reanimación & $7(100)$ & $18(26.9)$ & $11(11.1)$ & $23(3.5)$ \\
\hline $\begin{array}{l}\text { Oxígeno } \\
\text { suplementario }\end{array}$ & $7(100)$ & $66(98.5)$ & $86(86.9)$ & $261(40.2)$ \\
\hline SDR & $7(100)$ & $66(98.5)$ & $85(85.9)$ & $261(40.2)$ \\
\hline $\begin{array}{l}\text { Hemorragia } \\
\text { intraventricular }\end{array}$ & $0(0.0)$ & $2(3.0)$ & $0(0.0)$ & $1(0.3)$ \\
\hline Trauma neonatal & $0(0.0)$ & $1(1.5)$ & $1(1.0)$ & $4(1.1)$ \\
\hline Cefalohematoma & $0(0.0)$ & $0(0.0)$ & $0(0.0)$ & $1(0.1)$ \\
\hline Equimosis & $0(0.0)$ & $1(1.5)$ & $1(1.0)$ & $0(0.0)$ \\
\hline $\begin{array}{l}\text { Fractura de } \\
\text { clavícula }\end{array}$ & $0(0.0)$ & $0(0.0)$ & $0(0.0)$ & $1(0.1)$ \\
\hline Fractura de fémur & $0(0.0)$ & $0(0.0)$ & $0(0.0)$ & $1(0.3)$ \\
\hline Edema frontal & $0(0.0)$ & $0(0.0)$ & $0(0.0)$ & $1(0.3)$ \\
\hline Muerte neonatal & $6(85.7)$ & $20(29)$ & $5(5.0)$ & $4(0.6)$ \\
\hline
\end{tabular}

cesárea comparados con el parto vaginal, aunque los mecanismos no están claros. El aumento de la morbilidad puede estar relacionado con el impacto del nacimiento por cesárea en la adaptación respiratoria del recién nacido. Así, la decisión de realizar una cesárea en los pretérmino de más de 31 semanas debe tomarse con cautela y solo debe realizarse por condiciones obstétricas, no por la prematuridad (14).

En un estudio retrospectivo de 2.466 nacidos con peso inferior a 1.500 gramos de peso al nacer, proceden-

En Colombia, el Departamento Administrativo Nacional de Estadística (DANE) señala que el $11.4 \%$ de los nacimientos son $\mathrm{PP}$, lo cual corresponde a aproximadamente 100.000 niños pretérminos nacidos anualmente (12). En la Clínica de Maternidad Rafael Calvo, Cartagena-Colombia en el año 2011 , según informe hospitalario el $12.1 \%$ de los nacimientos fueron antes de las 37 semanas. Para el 2014 se presentaron 8.733 nacimientos, de los cuales el 1224 fueron PP, lo cual corresponde al $14.0 \%$ (datos no publicados). En Norteamérica, el problema se ha agravado, pues no solo se ha incrementado en el $10 \%$ de los PP, sino que además el índice de cesáreas en este grupo ha aumentado el 36\%. En los últimos diez años, el número de cesáreas realizadas antes de las 37 semanas de gestación ha ascendido del 33 al $50 \%(5,7,13)$.

De los casos incluidos en el estudio, el $40.9 \%$ de los neonatos requirieron ingreso a la unidad de cuidados intensivos. Cuando se observan por grupos según vía del parto brindada, el $52.9 \%$ de los recién nacidos por cesárea requirieron ingreso a la unidad, mientras que el $28.3 \%$ de los nacidos también lo ameritaron por vía vaginal, diferencia que es estadísticamente significativa.

Se ha señalado mayor riesgo de morbilidad y mortalidad perinatal entre los nacidos por tes de gestaciones simples en presentación cefálica, en ellos se evaluó la mortalidad y morbilidad neonatal y reportaron ligero aumento de distrés respiratorio aunque no significativo OR:1.2 [IC95\%:0.99-1.51]) en los nacidos por cesárea (15). En el presente estudio se observó diferencia significativa en cuanto a necesidad de ventilación mecánica y días de uso, siendo más favorable el parto vaginal. No se encontró diferencia significativa en cuanto a taquipnea transitoria del recién nacido, membrana hialina o hemorragia intraventricular, esta última es un hallazgo ecográfico extensamente estudiado en relación con ciertos factores obstétricos.

Existe controversia acerca de la vía del parto y el potencial daño neurológico en el recién nacido de bajo peso. Los nacidos vaginales por debajo de 1.500 gramos de peso tenían mayor riesgo de hemorragia intraventricular en comparación con los nacidos por cesárea OR: 2.5 [IC95\%:1.4-3.3]. El riesgo se incrementaba en $39 \%$ si el trabajo de parto duraba más de doce horas, en los nacidos por cesárea, el antecedente de trabajo de parto previo a la práctica de la cesárea incrementó el riesgo en $50 \%$. Existe aumento en el riesgo de hemorragia intraventricular en presencia de corioamnionitis y se ha sugerido que el riesgo de hemorragia intraventricular en PP está incrementado independientemente de la vía del parto (16-23). 
ISSN: 2215-7840, 6(2), julio-diciembre 2015, Beracaza-Robledo Cristina, Salcedo-Ramos Francisco, Rodríguez-Yance Benjamín,

Castañeda-Alfonso Sergio, Olarte-Parra Juan

Se ha propuesto cesárea de rutina en PP con el supuesto que al evitar el trabajo de parto se debería mejorar la supervivencia neonatal al reducir la hipoxia fetal, el trauma intracraneal y la hemorragia intraventricular. No obstante, la afirmación no ha sido demostrada (17). Algunos autores han concluido que la cesárea estaría indicada entre las 26-31 semanas de gestación con fetos en presentación cefálica (18), sin embargo, otros no han demostrado que la vía del parto influya en el resultado perinatal.

En un estudio, (10) al evaluar hemorragia intraventricular severa en nacidos con peso inferior a 1.250 gramos se observaron tasas mayores en nacidos por parto vaginal. Se han señalado como factores condicionantes para hemorragia intraventricular: la hipertensión arterial materna, insuficiente administración de corticoides prenatales, nacimiento del neonato con Apgar inferior a tres y sometido a maniobras de resucitación en el momento del parto (19).

Se presentaron seis casos de trauma obstétrico, cuatro en el grupo de parto vaginal (uno de fractura de clavícula, un cefalohematoma y dos con equimosis generalizadas) que corresponden al 1.5\%. Se presentaron dos casos en el grupo de cesárea (uno de fractura de fémur y uno de edema frontal) que son el $0.7 \%$. Las lesiones que pueden ocurrir en cesárea traumática incluyen: fracturas, daño nervioso periférico, lesión de médula espinal y hematoma subdural. El parto vaginal en presentación podálica en gestaciones pretérmino, se asocia con mayor riesgo de traumatismo obstétrico y de prolapso de cordón umbilical que en presentación cefálica (20). No ha sido posible demostrar que la vía del parto influya en el trauma neonatal (8).

Con respecto a la infección neonatal se encontró en el $29.4 \%$ de los nacidos por cesárea y el $11.4 \%$ de los nacidos vaginales, diferencia estadísticamente significativa. Wolf señaló en su estudio que no hubo diferencia significativa en la tasa de infecciones neonatales pero sí en la necesidad de soporte ventilatorio (9). La revisión de Cochrane del año 2008 (8) no obtuvo información suficiente para determinar la diferencia en la ocurren- cia de infección neonatal luego de parto vaginal frente a cesárea en PP.

En las historias clínicas de los 822 pacientes incluidos en el estudio se encontraron reportes de 17 muertes neonatales en el grupo de cesárea y 18 en el grupo de parto vaginal, lo cual corresponde al $2.99 \%$ y $4.54 \%$, respectivamente. No se encontraron diferencias significativas entre los dos grupos. El argumento que la cesárea electiva disminuye el riesgo de morbilidad y mortalidad neonatal a largo plazo se opone al argumento de que la práctica de una cesárea electiva conlleva un aumento de la morbilidad materna (10).

Existen pocos estudios que comparan de forma randomizada la vía abdominal vs. el parto vaginal en los casos de PP. Además, la evidencia disponible no apoya la recomendación de la práctica rutinaria de cesárea con la finalidad de mejorar la supervivencia o disminuir la morbilidad en los pretérminos extremos $(10,14)$.

Kitchen y cols. evaluaron 326 recién nacidos pretérminos entre las 24-28 semanas de gestación en relación a la vía del parto y el resultado neonatal a los dos años. La supervivencia a los dos años de toda la población fue del $52.8 \%$, siendo del $67.2 \%$ en los nacidos por cesárea comparado con el $50.9 \%$ de los nacidos vía vaginal, es decir, la diferencia no fue estadísticamente significativa. Además, la tendencia favorable hacia la cesárea no se confirmó después de realizar un análisis teniendo en cuenta los factores de confusión (17).

En el presente estudio no se encontró diferencia significativa en mortalidad neonatal en PP según la vía del parto, lo cual es acorde con lo presentado por diversos estudios (21-24). Existen varias consideraciones a la hora de decidir la vía del parto en los casos de gestaciones pretérmino: [A] vitalidad del feto, $[B]$ número de fetos, $[C]$ peso estimado fetal, [D] situación y presentación fetal y [E] edad gestacional (25). La mortalidad neonatal es aún elevada en PP, a pesar de la mejoría de la asistencia perinatal con valores globales del $4-6 \%$ con una gran variación dependiente del grado de prematuridad (22). 
A medida que se alcanza mayor edad gestacional se reduce la mortalidad perinatal y neonatal. Los pretérminos tardíos aportan el $10 \%$ y por tanto, también incrementan la mortalidad infantil. Las muertes perinatales de los pretérminos tardíos son tres veces más que la de los de término. En un estudio realizado en Utah-Estados Unidos, se encontró que los NP y pequeños para la edad gestacional tenían 44 veces más probabilidad de morir que los niños a término durante el primer mes (26). En los pretérmino extremos la mortalidad es superior al $90 \%$ y desciende conforme avanza la edad gestacional al nacer, es decir, mortalidad del $13 \%$ a las 28 semanas e inferior al $7 \%$ entre las 29 y 30 semanas, en un estudio realizado en España (27).

La limitación de este estudio fue su corte retrospectivo puesto que era necesario evaluar historias clínicas no elaboradas por los autores, lo cual es una importante fuente de sesgos. No se realizó análisis estadístico adecuado para establecer asociaciones entre las diferentes variables tomadas en consideración. Tiene como fortaleza ser el primer estudio de este tipo en la institución de re- ferencia y el primero en realizar un acercamiento inicial a la problemática. Los hallazgos deben ser reabordados, no permiten extrapolaciones y tampoco recomendaciones para el uso de la cesárea como la mejor vía de nacimiento para prevenir mortalidad perinatal en NP, ya que no ha demostrado tener ventajas sobre el parto vaginal (21-24).

\section{CONCLUSIÓN}

Los resultados perinatales adversos más prevalentes en NP por cesárea fueron: la necesidad de oxígeno suplementario, el síndrome de distrés respiratorio, el ingreso a UCIN, la infección neonatal, la sepsis temprana, la taquipnea transitoria y la enfermedad de membrana hialina. En NP por vía vaginal las morbilidades más prevalentes observadas fueron: el distrés respiratorio, taquipnea transitoria, sepsis e infección neonatal.

CONFLICTOS DE INTERESES: ninguno que declarar.

FINANCIACIÓN: recursos propios de los autores.

\section{REFERENCIAS BIBLIOGRÁFICAS}

1. Organization WH. International stadistical classification of diseases and related health problems. 10th ed: World Health Organization; 2012. p. 201.

2. Petrou S. The economic consequences of preterm birth during the first years of life. BJOG. 2005;112:10-5.

3. Petrou S, Mehta Z, Hockley C, Cook-Mozaffari P, Henderson J, Goldacre M. The impact of preterm birth on hospital in patient admissions and costs during the first 5 years of life. Pediatrics. 2003;112:1290-7.

4. Fainete P. Parto pretérmino: reto, reacciones y paradigmas. Rev Obstet Ginecol. 2012;72(4):21720.

5. Muglia L, Katz M. The enigma of spontaneous preterm birth. N Engl J Med. 2010;362:529-35.

6. Salcedo F, Rodríguez B, Borre O. Guía perinatal. 1er ed. Editorial Universidad de Cartagena; 2010.

7. Fleischman A, Iams J. Understanding and preventing preterm birth: the power of collaboration. Clin Perinatol. 2011;38(3):17-8.

8. Grant A, Glazener C. Cesarea electiva versus abordaje expectante para el parto de un bebe pequeño. Cochrane Lybrary. 2008;4.

9. Wolf H, Schaap A, Bruinse H, Smolders-de Haas H, Van Ertbruggen I, Treffers P. Vaginal delivary compared with cesarean section in early preterm breech delivery: a comparison of long term outcome. B J Obstet Gynecol. 1999;106:486-91.

10. Deulofeut $R$, Sola A, Lee B, Buchter S, Rahman M, Rogido M. The impact of vaginal delivery in premature infants weighing less than 1,251 grams. Obstet Gynecol. 2005;105(3):525-31.

11. Hogberg $U$, Holmgren P. Infant mortality of very preterm infants by mode of delivery, institucional policies and maternal diagnosis. Acta Obstet Gynecol. 2007;86:693-700.

12. http://www.dane.gov.co/index.php/esp/poblacion-y-demografia/nacimientos-ydefunciones/118-demograficas/estadisticas-vitales/2871-nacimientos-2011-preliminar.

13. Alfirevic Z, Milan S, Livio S. Caesarean section versus vaginal delivery for preterm birth in singletons (Review). Cochrane Database of Systematic Reviews. 2013;9.

14. Malloy M. Impact of cesarean section on intermediate and late preterm births: United States, 200-2003. Birth. 2009;36(1):26-33. 
ISSN: 2215-7840, 6(2), julio-diciembre 2015, Beracaza-Robledo Cristina, Salcedo-Ramos Francisco, Rodríguez-Yance Benjamín, Castañeda-Alfonso Sergio, Olarte-Parra Juan

15. Wylie B, Davidson L, Batra M, Reed S. Method of delivery and neonatal outcome in very lowbirthweight vertex-presenting fetus Am J Obstet Gynecol. 2008;198:289.e1-.e6.

16. Llurba E, Cabero L. Aistencia al parto prematuro. In: Cabero L, editor. Parto prepaturo. 1ra ed. Madrid: Editorial Medica Panamericana; 2004. p. 197-207.

17. Kitchen W,Ford GW,Doyle LW et al. Cesarean section or vaginaly at 24 to 28 weeks gestation: comparison of survival and neonatal and two-year morbidity.Obstet Gynecol1985;66(2):149-57.

18. Malloy M, Onstad L, Wright E. The effect of cesarean delivery on birth outcome in very low birth weight infants. Obstet Gynecol. 1991;77(4):498-503.

19. Riskin A, Riskin-Mashiah S, Bader D, Kugelman A, Lerner-Geva L, Boyko V, et al. Delivery mode and severe intraventricular hemorrhage in single, very low birth weight, vertex infants. Obstet Gynecol. 2008;112(1):21-8.

20. J. Monleón, J. Monleón Sancho, J. Mínguez Milio, J. Cervera, F. Morcillo, J. Mínguez. Elección de la vía del parto del gran pretérmino Hospital Universitario La F. Centro Maternal. Valencia. XVII Congreso Español de Medicina Perinatal.

21. Qiu H, Paneth N, Lorenz J, Collins M. Labor and delivery factors in brain damage, disabling cerebral palsy, and neonatal death in low-birth-weight infants. Am J Obstet Gynecol. $2003 ; 189(4): 1143-9$.

22. Skupski D, Greenough A, Donn S, Arabin B, Bancalari E, Vladareanu R. Delivery mode for the extremely premature fetus: a statement of the prematurity working group of the World Association of Perinatal Medicine. J Perinat Med. 2009;6:583-6.

23. Bittar R,Zugaib M. Qual é a melhor via de parto para o feto prematuro? Femina. 2010;38(10):

24. Vimercati A, Scioscia m, Nardelli C, Panella E, Laforgia N, Decosmo L, et al. Are active labour and mode of delivery still a challenge for extremly low birth weight infants? Experience at a tertiary care hospital. Eur J Obstet Gynecol Reprod Biol. 2009;145(2):154-7.

25. Cruz M, Caño A, González O, MFrenesda M. XVI Curso de actualización en obstetricia y ginecología. Vía del parto y operatoria obstétrica en el parto pretérmino; Granada, España: Hospital Universitario Virgen de las Nieves; 2012.

26. Pulver L, Guest-Warnick G, Stoddard G, Byington C, Young P. Weight for gestational age affects the mortality of late preterm infants. Pediatrics. 2009;123(6):1072-1077.

27. Rellan Rodríguez S, García de Ribera C, Aragón García P. El recién nacido prematuro. Protocolos Diagnóstico Terapeúticos de la AEP: Neonatología 2008.

Es el órgano de información científica de la Facultad de Medicina de la Universidad de Cartagena. Colombia.

Publique su trabajo en esta revista enviando su manuscrito a: revistacienciasbiomedicas@unicartagena.edu.co www.revistacienciasbiomedicas.com www.revistacienciasbiomedicas.com.co

Revista ciencias Biomédicas es una publicación independiente, imparcial, abierta, revisada por pares, de elevada visibilidad internacional, con circulación online e impresa. Publica artículos en todas las modalidades universalment aceptadas en inglés y en español, de temas referentes con todas las ciencias biomédicas, incluyendo ámbitos clínicos, epidemiológicos o de estudios básicos.

El sistema de gestión de manuscritos es rápido y justo.

Revista Ciencias Biomédicas está incluida en varias bases de datos latinoamericanas e internacionales.

Antes de enviar su manuscrito, revise las recomendaciones para los autores, presentes en www.revistacienciasbiomedicas.com www.revistacienciasbiomedicas.com.co 